\title{
Molecular dynamics simulations of liquid-liquid interfaces in an electric field: The water-1,2-dichloroethane interface
}

\author{
Cite as: J. Chem. Phys. 153, 164714 (2020); doi: 10.1063/5.0027876 \\ Submitted: 31 August 2020 - Accepted: 14 October 2020 • \\ Published Online: 28 October 2020
}

Paolo Raiteri, ${ }^{\text {a) }}$ (D) Peter Kraus, (D) and Julian D. Gale (D)

AFFILIATIONS

Curtin Institute for Computation, School of Molecular and Life Sciences, Curtin University, P.O. Box U1987, Perth, WA 6845, Australia

a) Author to whom correspondence should be addressed: p.raiteri@curtin.edu.au

\begin{abstract}
The polarized interface between two immiscible liquids plays a central role in many technological processes. In particular, for electroanalytical and ion extraction applications, an external electric field is typically used to selectively induce the transfer of ionic species across the interfaces. Given that it is experimentally challenging to obtain an atomistic insight into the ion transfer process and the structure of liquid-liquid interfaces, atomistic simulations have often been used to fill this knowledge gap. However, due to the long-range nature of the electrostatic interactions and the use of 3D periodic boundary conditions, the use of external electric fields in molecular dynamics simulations requires special care. Here, we show how the simulation setup affects the dielectric response of the materials and demonstrate how by a careful design of the system it is possible to obtain the correct electric field on both sides of a liquid-liquid interface when using standard 3D Ewald summation methods. In order to prove the robustness of our approach, we ran extensive molecular dynamics simulations with a rigid-ion and polarizable force field of the water/1,2-dichloroethane interface in the presence of weak external electric fields.
\end{abstract}

Published under license by AIP Publishing. https://doi.org/10.1063/5.0027876

\section{INTRODUCTION}

Liquid-liquid interfaces play a central role in a number of processes ranging from heterogeneous catalysis to, liquid chromatography, and heavy metal extraction to electrochemistry, and they have been the focus of intense research for more than half a century. ${ }^{1-4}$ In particular, polarized ITIES (Interfaces between Immiscible Electrolyte Solutions) have been widely used in sensing and bioanalytical chemistry. ${ }^{5}$ Although several different combinations of immiscible solvents can be prepared, the water-1,2-dichloroethane (DCE) is arguably the most commonly used and studied system. Intense efforts have been made to understand the molecular structure of the interface, which provided evidence to support either the old capillary wave theory for the structure of the interface ${ }^{6-8}$ or the existence of a mixed solvent layer. ${ }^{9-11}$ For most practical and technological applications though, it is the distribution and behavior of charged particles at the interfaces that are of utmost importance. For example, in the case of electrochemical applications, the accumulation of ions at the interfaces under the influence of an applied electric field contributes to the formation of an interface potential, which regulates the transfer of ions from one phase to the other. Because of the difficulties in probing soft interfaces, the description of the distribution of electrolytes at liquid-liquid interfaces has often been based on mean field theories developed for solid-liquid interfaces, such as the Gouy-Chapman or the Stern double-layer model.

Although experimental techniques, such as time-resolved fluorescence spectroscopy, ${ }^{12}$ second harmonic generation spectroscopy, ${ }^{13}$ and $\mathrm{x}$-ray scattering, ${ }^{14,15}$ have had some success in providing information on the structure of the interfacial region, computer simulations have been the only tool that could directly provide a molecular picture of the structure of liquid-liquid interfaces. Molecular Dynamics (MD) has been the method most commonly used in computational studies of liquid-liquid interfaces due to its ability to provide both thermodynamic and kinetic data for the simulated systems. Since the pioneering work of Benjamin on the 
water-DCE interface, ${ }^{16-18}$ MD simulations have been widely used to study the structure of the interface, ${ }^{8,19}$ ion transfer, ${ }^{20-24}$ and ion distributions with and without external electric fields. ${ }^{25-27}$ However, the predictive power of MD simulations is strongly dependent on the accuracy of the simulation protocols used. This includes both how the atomic interactions are described and how the simulations are designed, particularly in the case of polarized interfaces where the presence of an external field is often incompatible with standard simulation protocols.

The use of an electric field in MD has not been unique to liquidliquid interfaces but has also been applied to polarize membranes and to induce an ion flux through pores and nano-tubes. However, the standard approach that is often used is to add a force proportional to the atoms' partial charges, but little attention is paid to whether this is compatible with the choice of boundary conditions, i.e., $3 \mathrm{D}$ periodic or slab geometry, and the treatment of the long range electrostatics. Moreover, to the best of our knowledge, there seems to be a complete lack of characterization of whether the dielectric response of the materials is consistent with the macroscopic behavior of dielectrics.

In the rest of this paper, we will discuss the constraints imposed on the simulation setup by the use of 3D Ewald summation methods to treat the long range electrostatics and how this limits the applicability of external electric fields to MD. We will then propose and validate a method to correctly include an electric field in 3D periodic MD simulations, which is compatible with the standard Ewald summation framework, and apply it to the water/DCE interface.

\section{A. Ewald summation and electric fields}

Even with modern supercomputers, MD simulations are limited to a relatively small number of atoms when compared to the macroscopic size of bench-top experiments, and since the inception of MD many techniques have been introduced to speed up such calculations and reduce both surface and finite size effects (see, e.g., Ref. 28). Most of these procedures are nowadays so commonly used that little consideration is often given to their side effects, particularly by the inexperienced practitioner. In particular, the vast majority of MD simulations employ 3D periodic boundary conditions and a short-range cutoff for the non-bonded interactions, which require the simulation cell to be at least twice as large as the cutoff distance (i.e., they obey the minimum image convention). Although this combined approach has the advantage of eliminating all free surfaces and vastly reducing the computational cost of the pairwise interactions, results from MD simulations still suffer from finite size effects, and in certain instances, the results have been shown to depend on the cutoff distance. Cases in point are the calculation of the self-diffusion coefficient and the surface tension. $^{29,30}$

Because of the long-range nature of the Coulomb interaction, where the rate of decay is less than the increase in the radial density of charges, it was recognized early on that a short-range cutoff for the electrostatic interactions would have led to unphysical results. A variety of schemes have been developed throughout the years to efficiently compute the long-range electrostatic interactions in MD simulations. The Ewald summation (including accelerated variants) is by far the most widely used method, and although a complete description of this method is beyond the scope of the present work, it is important to briefly highlight the basic ideas and assumptions behind it. Within the framework of periodic boundary conditions, any simulated system effectively becomes infinite and 3D periodic, and this allows for rewriting the conditionally convergent sum of the electrostatic pairwise interactions as the sum of a finite real space term, through the addition of Gaussians of opposite sign to the point charges, and compensation via a Fourier transform of this smooth charge density, leading to a rapidly converging term in reciprocal space. The former is simply a sum of pairwise interactions up to a cutoff distance, while the latter accounts for the long-range part of the electrostatics and involves a sum over the reciprocal space vectors of the system. The partitioning of the calculation between real space and reciprocal space terms is largely arbitrary and is primarily driven by efficiency arguments. Typically, the same cutoff radius is chosen for the van der Waals and electrostatic interactions, and depending on the system size, different amounts of computational work are done in reciprocal space. In most modern codes, the number of reciprocal space vectors used in the Ewald summation is typically automatically determined based on one single input parameter that represents the desired accuracy of the calculation. While this is generally well-known, the split of the electrostatic interactions into real and reciprocal space terms does not automatically make the answer well-defined, and further conditions have to be specified to lead to a consistent result. In particular, it is necessary to make an assumption regarding the macroscopic dielectric properties of the system beyond the cutoff radius. The most common assumption is the so called "tin-foil" approximation, which assumes that the system beyond the cutoff radius is a conductor $\left(\varepsilon_{r}=\infty\right)$, and it requires that there is no net charge in the simulation cell and no dipole across the entire system (Fig. 1). Therefore, the result of the Ewald sum is directly linked to assumptions regarding the macroscopic state of the system, including the surfaces, when comparing to real materials. Despite most systems not being conducting materials, the "tin-foil" approximation has several benefits, such as that the energy is independent of the origin, and it is conserved when charged particles cross the simulation boundaries. If either of those two conditions is not met, correction terms need to be added to eliminate any artifacts caused by the departure from the limit of the "tin-foil" approximation. ${ }^{31,32}$ This is particularly problematic for MD simulations with a slab geometry where the artificial periodicity in the direction normal to the slab imposed by the 3D boundary conditions has been shown to cause artifacts. ${ }^{33}$ A typical approach

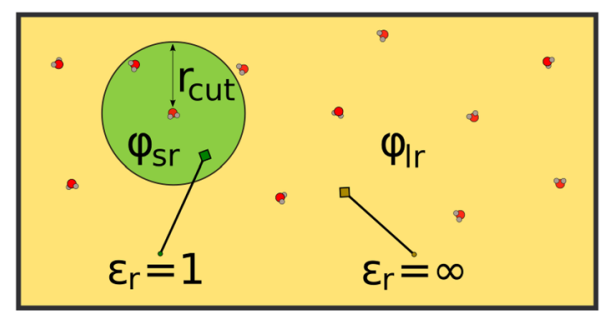

FIG. 1. Schematic representation of the "tin-foil" approximation used in the most common implementation of the Ewald summation, $\phi_{s r}$ and $\phi_{\text {Ir }}$ represent the real space and reciprocal space contributions to the Ewald summation, respectively. 
for simulating interfacial problems with $3 \mathrm{D}$ boundary conditions is to add a region of vacuum above the slab and assume that there is no interaction between the periodic images. However, $\mathrm{Spohr}^{34}$ showed that the results of the 3D Ewald summation converge very slowly to the correct $2 \mathrm{D}$ results, and even for a cell 5 times larger in the direction normal to the slab to the in-plane dimensions, the agreement was not satisfactory; a geometry that is very rarely seen in the literature.

There exists a lesser-used alternative limit to the tin-foil boundary conditions for the Ewald sum in which the dielectric constant at the cutoff boundary is assumed to have a finite value. In this case, a correction to the standard Ewald energy arises based on the total macroscopic dipole averaged per unit cell,

$$
U^{\text {surf }}=\frac{2 \pi}{\left(2 \varepsilon_{\text {surf }}+1\right) V}\left[\left(\sum_{i} q_{i} \mathbf{r}_{i}\right)^{2}+2\left(\sum_{i} q_{i} \mathbf{r}_{i}\right) \cdot\left(\sum_{j} \mathbf{p}_{j}\right)+\left(\sum_{i} \mathbf{p}_{i}\right)^{2}\right],
$$

where $\mathbf{r}, q$, and $\mathbf{p}$ are the atomic positions, charges, and dipoles, respectively, and $\varepsilon_{\text {surf }}$ is the value of the dielectric constant at the cutoff boundary, which is often assumed to be equal to 1 .

The use of this expression is problematic since the dipole moment is intrinsically ill-defined under periodic boundary conditions since the value depends on the choice of image used for each atom. Hence, it is only typically used to define the contribution from changes in polarization rather than the absolute value. When considering the specific case of a $3 \mathrm{D}$ simulation of what is intended to be a $2 \mathrm{D}$ problem (i.e., a slab model with vacuum gaps), then the net dipole in the direction of the surface normal can now be defined unambiguously since the surfaces are explicitly included in the simulation. Based on this, Yeh and Berkowit ${ }^{32}$ proposed the $1 \mathrm{D}$ version of this correction term as a means of correcting the results to match those where open boundary conditions are present in one direction. This corrects the Ewald sum result from 3D to give results that match those from the 2D Parry summation. ${ }^{36,37}$ Despite this theory having been established many decades ago and artifacts due to the use of the Ewald summation in $3 \mathrm{D}$ periodic systems having been reported extensively in the literature (see, e.g., Refs. 33, 38, and 39) because the necessary correction terms are either not available in the code of choice or have simply been ignored, the literature is riddled with papers that potentially contain serious errors due to an erroneous treatment of the long range part of the electrostatic interactions.

The use of external electric fields in MD simulations with 3D Ewald summation methods is therefore even more problematic, because of the permanent dipoles that are induced in the simulation cell. The naïve assumption, which is however commonly found in the literature, that the presence of an external electric field in MD simulations is equivalent to adding a force proportional to the atomic charges throughout the system is indeed too simplistic. First of all, it violates the assumption behind the "tin-foil" approximation that there is no net dipole in the system. Moreover, it cannot account for the fact that in inhomogeneous systems the strength of the electric field can be different in different parts of the simulation cell, e.g., on the opposite sides of a liquid-liquid interface.

In this work, we propose a way to design $3 \mathrm{D} \mathrm{MD}$ simulations with an external electric field that reproduces the expected behavior of dielectric materials and show that the artifacts present in other approaches can be mitigated even when using a standard 3D Ewald summation method. This involves the use of four charged plates and two slabs of atoms arranged in an anti-symmetric fashion along the direction of the electric field, which by design cancels any internal dipoles, on average, and allows the use of 3D periodic Ewald summation methods, therefore leading to efficient MD simulations (Fig. 2). It is worth noting here that the use of genuinely $2 \mathrm{D}$ summation methods, such as the one developed by Parry mentioned above, would avoid all the complications and associated an increase in computational cost of setting up larger symmetric MD simulations, but because it is not widely implemented in the commonly used MD codes, the method proposed here can be more readily applied. Moreover, our method has the further advantage of doubling the statistics by running two replicas of the same system at once.

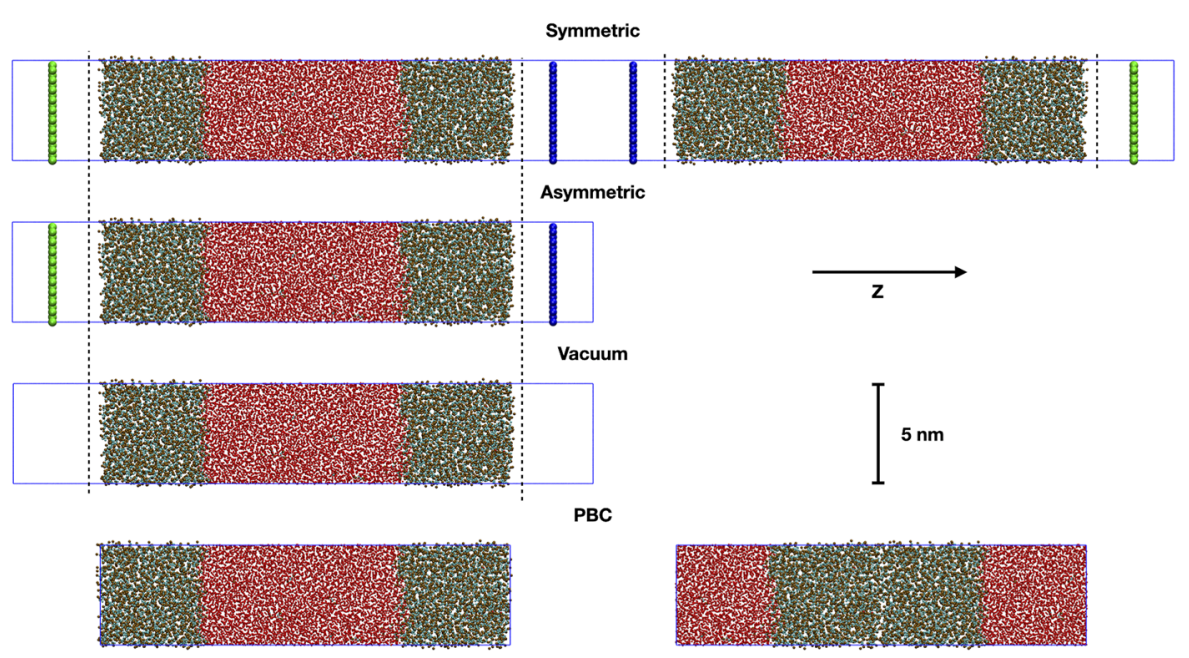

FIG. 2. Example of the system geometry used in the simulations with an external electric field. The oxygen, carbon, and chlorine atoms are colored in red cyan, and ochre, respectively. Hydrogen atoms have been removed for clarity. The green and blue spheres represent the positive and negative charges that make up the plates of the capacitor used to create the external electric field. The vertical dashed lines show the approximate positions of the restraining walls used to confine the liquids. The blue rectangles show the size of the simulation boxes used; for the "vacuum" and "PBC" setups, we also tested different lengths in $z$. 


\section{THEORETICAL METHODS}

Although for practical and technological applications, neat liquids are of limited interest, they still represent a significant challenge to our molecular understanding of the structure of liquid-liquid interfaces, particularly in the presence of external electric fields. In this study, we will focus on the water to 1,2-dichloroethane (DCE) interface, which obviously requires appropriate force fields for both individual liquids, as well as the cross-interaction. While there are numerous existing force fields for water, far fewer have been derived specifically for DCE. Here, we have performed MD simulations with a rigid-ion force field taken from the literature ${ }^{40}$ and with a new polarizable force field developed as part of this work, which was based on the AMOEBA model and includes a multipole expansion of the atomic charges up to the quadrupole in addition to atomic polarizability.

For the development and testing of the water/DCE force field, a variety of different codes and tools have been used, and below, we provide a short description of the methods employed. The primary computational tool used in this work was MD, and three different codes have been used: LAMMPS ${ }^{41}$ for all the simulations with the rigid-ion model, TINKER HP v1.2 $2^{42}$ for the solvation free energy calculations with the AMOEBA polarizable force field, and openMM ${ }^{43,44}$ for all other calculations with the AMOEBA force field. All production MD simulations were performed with a $1 \mathrm{fs}$ time step in the NVT ensemble at $300 \mathrm{~K}$ with no constraints on the covalent bonds and angles, and the atomic coordinates were stored every 1 ps. The temperature was controlled with a chain of Nosé-Hoover thermostats of length 5 and a relaxation time of 1 ps. For the bulk liquid simulations, the cell density was initially equilibrated in the NPT ensemble at 1 atm using an isotropic or semi-isotropic barostat for the pure liquids and interface simulations, respectively. For the simulations with a slab in vacuum, harmonic walls were placed around the slabs to prevent the system from drifting and the liquid molecules from evaporating. The positions of the walls were such that they normally did not interfere with the atoms unless they had evaporated from the slab. In this case, a Langevin thermostat was also used to limit the drift of the slabs. In the case of the AMOEBA polarizable force field, the atomic induced dipoles were determined self-consistently at every MD step with the convergence tolerance set to $10^{-5}$.

\section{A. Force field parameters}

The rigid-ion force field used in this work for DCE is based on the Generalized Amber Force Field ${ }^{45}$ (GAFF) model, and it was taken from the literature. ${ }^{40}$ The $\mathrm{SPC} / \mathrm{Fw}^{46}$ model was instead used to describe water. The general form of the interaction potential can be separated in the classical intramolecular forms for bond stretching, angle-bending, and torsions,

$U_{\text {intra }}=k_{b}\left(b_{i j}-b_{0}\right)^{2}+k_{\theta}\left(\theta_{i j k}-\theta_{0}\right)^{2}+k_{\phi}\left[1+\cos \left(n \phi_{i j k l}-\phi_{0}\right)\right]$,

while the intermolecular contributions are the sum of the Coulomb term and a Lennard-Jones potential,

$$
U_{\text {inter }}=\frac{1}{4 \pi \varepsilon_{0}} \frac{q_{i} q_{j}}{r_{i j}}+4 \varepsilon_{i j}\left[\left(\frac{\sigma_{i j}}{r_{i j}}\right)^{12}-\left(\frac{\sigma_{i j}}{r_{i j}}\right)^{6}\right] .
$$

For the development of a polarizable force field for DCE, we started from the modified AMOEBA09 parameters ${ }^{47}$ and followed the standard procedure described in Ref. 48 . The molecular geometry of DCE was relaxed at the MP2/6-31G* level of theory, and the electrostatic potential around the molecule was computed using a larger basis set $\left(\mathrm{MP} 2 / 6-311 \mathrm{G}^{* *}\right)$. The multipole moments were then determined using a Gaussian distributed multipole analysis ${ }^{49}$ based on the poledit program that is included in the TINKER distribution. The atomic polarizabilities, intramolecular, and van der Waals parameters were obtained from similar molecules that were already included in the AMOEBA09 force field, and the standard AMOEBA combination rules were used for all the intermolecular cross-interactions. ${ }^{5}$

For all the simulations reported in this work, we set the accuracy of the Ewald summation to $10^{-5}$, but each code used different implementations of the method. In LAMMPS, we used the particleparticle particle-mesh Ewald, ${ }^{51,52}$ in TINKER, the smooth particle mesh Ewald, ${ }^{53}$ and in openMM, the particle mesh Ewald. ${ }^{54}$ All benchmark calculations with the 2D Parry summation method have been performed with the GULP program.

The SPC/Fw and AMOEBA09 water parameter sets were chosen for compatibility with our earlier works on calcium, carbonate, bicarbonate, and sulfate in water, ${ }^{57,58}$ which will be added to the liquid-liquid interface in a future work; a full characterization of these force fields will be given below. For completeness, the force field parameters used in this work are reported in the supplementary material.

\section{B. Quantum chemical calculations}

The quantum chemical calculations of dipole moments and relative energies of $\mathrm{H}_{2} \mathrm{O}$ and DCE in vacuum, $\mathrm{H}_{2} \mathrm{O}$, and DCE were performed with the Psi4 package (version 1.4a2.dev523). ${ }^{59}$ The PWPB95-D $3^{60}$ double-hybrid density functional approximation was selected due to its excellent performance for dipole moments of closed-shell species as recommended by Hait and Head-Gordon. ${ }^{61}$ The present results are obtained with the aug-pc-3 basis sets, ${ }^{62}$ which are diffusely augmented basis sets of approximately $4-\zeta$ quality. The calculated dipoles and energy differences from PWPB95-D3 are consistent with the $\mathrm{M} 06-2 \mathrm{X}^{63}$ single-hybrid density functional approximation, applied with aug-pc-3 as well as aug-pc-4 basis sets.

Calculations in $\mathrm{H}_{2} \mathrm{O}$ and DCE were performed using an implicit solvent model, as implemented in the PCMsolver package. ${ }^{64}$ The solver type was set to IEFPCM, with solvent parameters set to $\mathrm{H}_{2} \mathrm{O}$ or DCE as required, with the solvent cavity constructed using UFF radii, using GePol with an area of $0.3 \AA^{2}$. As analytic gradients or Hessians are not available in Psi4 when the PCMsolver is used, and evaluation of the PWPB95-D3/aug-pc-3 potential by doublefinite-differences would be too costly, the free energy corrections to the energy differences are evaluated using the significantly cheaper composite method PBEh-3c. ${ }^{65}$

\section{FORCE FIELD VALIDATION}

Before discussing the effect of an external electric field on the properties of the water/DCE interface, it is important to provide 
a careful characterization of the pure liquids and of the liquid vapor interfaces, particularly for the AMOEBA force field that was developed in this work where no prior data exist. To this end, we computed several properties of the pure liquids and of the binary system, and compared them with the available experimental data. In Table I, we report the properties of both liquids with regard to their density $(\rho)$, self-diffusion coefficient $\left(D^{\infty}\right)$, shear viscosity $(\eta)$, enthalpy of vaporization $\left(\Delta H_{v a p}\right)$, solvation free energy $\left(\Delta G_{s o l v}\right)$, surface and interfacial tensions $(\gamma)$, relative dielectric constant $\left(\varepsilon_{r}\right)$, interfacial width $\left(d_{0}\right)$, mutual solubility $(x)$, and average molecular dipole $(\langle\mu\rangle)$.

The self-diffusion coefficient $D^{\infty}$ was computed for three box sizes $(5 \mathrm{~nm}, 7.5 \mathrm{~nm}$, and $10 \mathrm{~nm}$ ) using the Einstein equation,

$$
D=\frac{1}{6}\left\langle|r(t)-r(0)|^{2}\right\rangle,
$$

and extrapolated to an infinite system using its well-known sizedependence, $^{29}$

TABLE I. Properties of the pure liquids. The uncertainties correspond to the standard error of the average or the uncertainty obtained from the least square fitting (see text for details).

\begin{tabular}{lccc}
\hline \hline Water & Expt. & Rigid-ion & AMOEBA \\
\hline$\rho\left(\mathrm{g} / \mathrm{cm}^{3}\right)$ & $0.997^{\mathrm{a}}$ & $0.998 \pm 0.01$ & $1.000 \pm 0.01$ \\
$D^{\infty}\left(10^{-5} \mathrm{~cm}^{2} / \mathrm{s}\right)$ & $2.3^{\mathrm{b}}$ & $2.8 \pm 0.01$ & $2.7 \pm 0.01$ \\
$\eta(\mathrm{mPa} \mathrm{s})$ & $0.89^{\mathrm{c}}$ & $0.67 \pm 0.08$ & $0.78 \pm 0.05$ \\
$\Delta \mathrm{H}_{\text {vap }}(\mathrm{kJ} / \mathrm{mol})$ & $44.0^{\mathrm{a}}$ & $48.7 \pm 0.1$ & $43.5 \pm 0.1$ \\
$\Delta \mathrm{G}_{\text {solv }}(\mathrm{kJ} / \mathrm{mol})$ & $-26.5^{\mathrm{d}}$ & $-28.3 \pm 0.1$ & $-24.8 \pm 5$ \\
$\gamma(\mathrm{dyn} / \mathrm{cm})$ & $72.14^{\mathrm{e}}$ & $63.5 \pm 1$ & $63 \pm 1$ \\
$\varepsilon_{r}$ & $80^{\mathrm{f}}$ & $80 \pm 0.1$ & $91 \pm 0.1$ \\
$d_{0}(\mathrm{~nm})$ & $\ldots$ & 0.4 & 0.4 \\
\hline $\mathrm{DCE}$ & & & \\
\hline$\rho\left(\mathrm{g} / \mathrm{cm}^{3}\right)$ & $1.24^{\mathrm{g}}$ & $1.19 \pm 0.01$ & $1.19 \pm 0.01$ \\
$D^{\infty}\left(10^{-5} \mathrm{~cm} / \mathrm{s}\right)$ & $1.69^{\mathrm{h}}$ & $2.4 \pm 0.01$ & $1.41 \pm 0.02$ \\
$\eta(\mathrm{mPa} \mathrm{s})$ & $0.727^{\mathrm{i}}$ & $0.62 \pm 0.08$ & $0.85 \pm 0.05$ \\
$\Delta \mathrm{H}_{\text {vap }}\left(\mathrm{kJ} / \mathrm{mol}^{2}\right)$ & $35.1^{\mathrm{j}}$ & $38.1 \pm 0.1$ & $38.2 \pm 0.1$ \\
$\Delta \mathrm{G}_{\text {solv }}\left(\mathrm{kJ} / \mathrm{mol}^{2}\right)$ & $-19.66^{\mathrm{k}}$ & $-18.4 \pm 0.1$ & $-19.0 \pm 1$ \\
$\gamma(\mathrm{dyn} / \mathrm{cm})$ & $32.57^{\mathrm{l}}$ & $37 \pm 1$ & $32 \pm 1$ \\
$\varepsilon_{r}$ & $10.7^{\mathrm{m}}$ & $19 \pm 0.1$ & $19 \pm 0.1$ \\
$d_{0}(\mathrm{~nm})$ & $\ldots$ & 0.8 & 0.8 \\
\hline \hline
\end{tabular}

${ }^{\mathrm{a}}$ From Ref. 66.

${ }^{\mathrm{b}}$ From Ref. 67.

${ }^{\mathrm{c}}$ From Ref. 68 .

${ }^{\mathrm{d}}$ From Ref. 69.

${ }^{\mathrm{e}}$ From Ref. 70 .

${ }^{\mathrm{f}}$ From Ref. 71.

${ }^{\mathrm{g}}$ From Ref. 72 .

${ }^{\mathrm{h}}$ From Ref. 73 .

${ }^{\mathrm{i}}$ From Refs. $72-74$.

${ }^{\mathrm{j}}$ From Ref. 75 .

${ }^{\mathrm{k}}$ From Ref. 69.

${ }^{1}$ From Ref. 70 .

${ }^{\mathrm{m}}$ From Ref. 76 .

$$
D_{P B C}=D^{\infty}-\frac{k_{B} T \xi}{6 \pi \eta L},
$$

where $\xi \approx 2.837297$ and $D_{P B C}$ indicates the self-diffusion coefficient computed in a box of finite size $L$. The slope of the best fitting line was then used to determine the shear viscosity of the fluids $(\eta)$.

The enthalpy of vaporization was computed using

$$
\Delta H_{\text {vap }}=\left\langle U_{\text {gas }}\right\rangle-\left\langle U_{\text {liq }}\right\rangle+R T,
$$

and the solvation free energy was obtained using the free energy perturbation procedure in which one of the molecules in the system was labeled a solute and its interactions with the solvent were progressively removed or added,

$$
\Delta G=-k_{B} T \ln \sum_{i}\left\langle\exp \left(-\Delta U\left(\lambda_{i}, \lambda_{i \pm 1}\right)\right)\right\rangle_{\lambda_{i}},
$$

where $0 \leq \lambda_{i} \leq 1$ is a scaling parameter that was used to progressively switch on or off the van der Waals and Coulomb interactions between this molecule and the solvent. $\langle\cdots\rangle_{\lambda_{i}}$ indicates a thermodynamic average computed during an MD simulation with the scaling parameter set to $\lambda_{i}$, and $\Delta U$ is the energy change when the scaling parameter is changed to $\lambda_{i+1}$ or $\lambda_{i-1}$ depending on whether the molecule is being created or annihilated, respectively, keeping the coordinates fixed. Each type of interaction was perturbed independently over 20 stages, and each stage was comprised of a 2 ns MD run. For a detailed description of the calculations, including a sensitivity study on the effects of the box size, ensemble, and direction of the perturbation, see the supplementary material.

The surface tensions of the liquid/vapor and liquid/liquid interfaces were computed using the following equation:

$$
\gamma=\frac{L_{z}}{2}\left[P_{z}-0.5\left(P_{x}+P_{y}\right)\right]
$$

where $L_{z}$ is the box dimension in the direction normal to the interface, $P_{i}$ are the components of the pressure tensor in the three Cartesian directions, and the $1 / 2$ is required because in our $3 \mathrm{D}$ periodic system, there are two interfaces. The surface tension was computed for three values of the real space cutoff distance $(1.1 \mathrm{~nm}, 1.5 \mathrm{~nm}$, and $2 \mathrm{~nm})$ and extrapolated to the infinite cutoff to account for its dependence on the inverse of the cutoff radius squared. ${ }^{3}$

The interface thickness was calculated by fitting the density profiles of one species along the $z$ axis with a sigmoidal function,

$$
\rho(z)=\frac{1}{2}\left(\rho_{1}+\rho_{2}\right)-\frac{1}{2}\left(\rho_{1}-\rho_{2}\right) \operatorname{erf}\left(\frac{z-z_{0}}{d_{0}}\right),
$$

where erf is the error function, $z_{0}$ is the position of the interface, $d_{0}$ is a parameter related to the interface thickness, and $\rho_{1}$ and $\rho_{2}$ are the equilibrium densities of the species on the opposite sides of the interface. In the case of a liquid/vapor interface, $\rho_{1}$ and $\rho_{2}$ correspond to the equilibrium densities in the liquid and vapor phases, respectively, while for the liquid/liquid interfaces, $\rho_{2}$ corresponds to the solubility of one species into the other liquid, and because the 


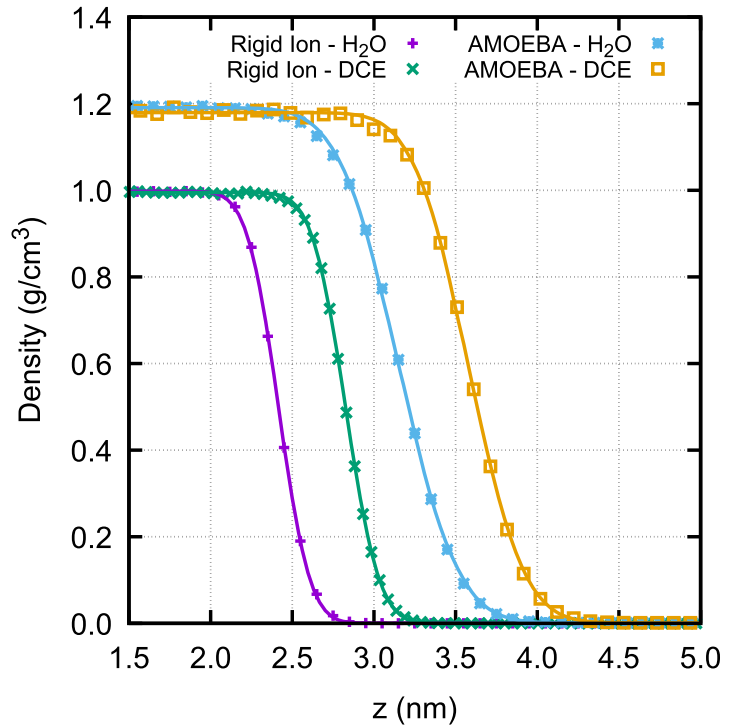

FIG. 3. Density profiles at the vapor interface for liquid water and DCE predicted by the rigid-ion and AMOEBA force fields (points). The lines are the best fit curves obtained using Eq. (9). The curves have been offset along $z$ for clarity.

liquids are largely immiscible, $\rho_{1}$ remains approximately the bulk density of the pure fluids. The interface thickness was then estimated as $1.8 d_{0}$, which corresponds to the range over which the density of the liquid drops from $90 \%$ to $10 \%$ of its bulk value (Fig. 3).

The dielectric constant of the liquids has been computed using the standard formula,

$$
\varepsilon_{r}=\varepsilon_{r}^{\infty}+\frac{\left\langle M^{2}\right\rangle}{3 \varepsilon_{0} V k_{B} T}
$$

where $\left\langle M^{2}\right\rangle$ is the average fluctuation of the total cell dipole, $\varepsilon_{0}$ is the vacuum permittivity, $k_{B}$ is the Boltzmann constant, and $T$ is the simulation temperature. For the AMOEBA force field, the highfrequency relative permittivity, $\varepsilon^{\infty}$, was then obtained using the Clausius-Mossotti formula,

$$
\frac{\varepsilon_{r}^{\infty}-1}{\varepsilon_{r}^{\infty}+2}=\frac{4 \pi}{3 V} \sum_{i} \alpha_{i},
$$

where $\alpha_{i}$ are the atomic polarizabilities in CGS units. ${ }^{78}$ The highfrequency dielectric constants were found to be 2 and 2.2 for water and DCE, respectively, which is consistent with the experimental estimate of the high-frequency dielectric permittivity of water.'

The average molecular dipole moment, $\langle\mu\rangle$, was computed for species in the liquid and gas phases and when dissolved in the other solvent (Table II). In the case of the AMOEBA force field, it included both the permanent and induced dipole terms. Due to the scarcity of experimental estimates of the molecular dipole for DCE, we compared our numbers with those estimated from QM calculations in vacuum and within an implicit solvent. Although this is not representative of the true molecular environment, particularly for water where hydrogen bonding is certainly important, it provides an indication of the direction and size of the change in the dipole moment. In the case of water, the simple QM calculations performed in this work with the PCM implicit solvent appear to underestimate the dipole moment of the water molecules in the liquid phase obtained from more sophisticated approaches, and we would therefore expect the same to occur for DCE. Moreover, the actual average dipole moment of DCE in the liquid phase depends on the relative fraction of molecules in the trans and gauche conformations (Table III).

\section{A. Pure liquids}

Overall both force fields perform well and very similarly in reproducing all the computed properties of the pure liquids, and all

TABLE II. Dipole moment of water and DCE computed using the force fields used in this work and the PWPB95-D3 functional. The three values shown for DCE correspond to the average dipole moment of the trans and gauche conformations and the ensemble average computed during the MD simulation with the rigid-ion and AMOEBA force fields or from the $0 \mathrm{~K}$ minimized structures with the PWPB95-D3 functional. Only one number is reported from the experimental values, and it is assumed to correspond to an ensemble average. All values are in Debye.

\begin{tabular}{lccccc}
\hline \hline Species & Phase & Literature & Rigid-ion & AMOEBA & PWPB95-D3 \\
\hline \multirow{2}{*}{ Water } & Gas & $1.80^{\mathrm{a}}$ & 2.40 & 1.80 & 1.90 \\
& Liquid & $2.90^{\mathrm{b}}$ & 2.40 & 2.75 & 2.29 \\
& Solute & $\ldots$ & 2.28 & 2.15 & 2.23 \\
\hline DCE & Gas & $1.1-1.8^{\mathrm{c}}$ & $0.5 / 3.8 / 1.6$ & $0.3 / 3.3 / 0.75$ & $0.0 / 2.77 /-$ \\
& Liquid & $1.6^{\mathrm{d}}$ & $1.9 / 3.8 / 3.0$ & $0.6 / 4.4 / 2.9$ & $0.0 / 3.81 /-$ \\
& & $0.77 / 3.6 / 2.7^{\mathrm{e}}$ & & & \\
& Aqueous & $1.17 / 4.50 / 3.1^{\mathrm{e}}$ & $0.5 / 3.8 / 3.3$ & $0.6 / 4.6 / 3.5$ & $0.0 / 4.01 /-$ \\
\hline \hline
\end{tabular}

${ }^{\text {a }}$ From Ref. 80.

${ }^{\mathrm{b}}$ From Ref. 81.

${ }^{c}$ From Ref. 82.

${ }^{\mathrm{d}}$ From Ref. 83.

${ }^{\mathrm{e}}$ From Ref. 84 . 
TABLE III. Free energy of the gauche conformation relative to the trans conformation for DCE in the gas phase, in the bulk liquid, and when dissolved in water (aqueous). $\Delta G^{*}$ is the free energy barrier for the trans to gauche rotation. The free energy as a function of the torsional angle has been computed as $-k_{B} T \ln [P(\theta)]$, where $P$ is the distribution of the $\mathrm{Cl}-\mathrm{C}-\mathrm{C}-\mathrm{Cl}$ torsion angle collected during the $\mathrm{MD}$ simulations (see the supplementary material). The fraction of molecules that are in the gauche conformation has been computed from the area under the gauche and trans peaks of the probability distribution. For the PWPB95-D3 calculations, the free energy was computed with thermodynamic corrections at $300 \mathrm{~K}$, and the numbers in brackets are the $0 \mathrm{~K}$ energy differences.

\begin{tabular}{|c|c|c|c|c|}
\hline & & $\Delta G(\mathrm{~kJ} / \mathrm{mol})$ & $\begin{array}{l}\text { Molar } \\
\text { fraction }\end{array}$ & $\Delta G^{*}(\mathrm{~kJ} / \mathrm{mol})$ \\
\hline \multirow[t]{3}{*}{ Rigid-ion } & Gas & +3.5 & 0.30 & 5.9 \\
\hline & Liquid & -2.1 & 0.77 & 4.8 \\
\hline & Aqueous & -3.4 & 0.85 & 4.6 \\
\hline \multirow{3}{*}{ AMOEBA } & Gas & +6.7 & 0.10 & 18.9 \\
\hline & Liquid & +0.1 & 0.62 & 14.7 \\
\hline & Aqueous & -1.2 & 0.73 & 13.9 \\
\hline \multirow[t]{3}{*}{ Experiment $^{\mathrm{a}}$} & Gas & +4.6 & 0.23 & $11^{\mathrm{b}}$ \\
\hline & Liquid & -0.04 & 0.65 & $\ldots$ \\
\hline & Aqueous & $\ldots$ & $\ldots$ & $\ldots$ \\
\hline \multirow[t]{3}{*}{ PWPB95-D3 } & Gas & $+5.5(+5.7)$ & 0.10 & $19.9(19.1)$ \\
\hline & Liquid & $+0.7(+1.0)$ & 0.43 & $19.1(17.5)$ \\
\hline & Aqueous & $+1.2(-0.1)$ & 0.32 & $18.4(17.2)$ \\
\hline
\end{tabular}

${ }^{\mathrm{a}}$ From Ref. 85.

${ }^{\mathrm{b}}$ From Ref. 86.

the properties computed in this work agree with the values previously published for the water models. ${ }^{40,46,47,87,88}$ In the case of DCE, the AMOEBA force field appears to be slightly superior, with the major shortfall of the force fields being the prediction of the dielectric constant, which is off by almost a factor of two. It is worth mentioning that the values of the self-diffusion coefficient for water reported here appear to be larger than the literature values for both the AMOEBA09 and rigid-ion force fields. Possible reasons for this discrepancy could be the lack of finite size corrections or the use of a Langevin thermostat in the original papers for the rigid-ion and AMOEBA09 force fields, respectively.

Significant differences between the rigid-ion and AMOEBA force fields appear when looking at the molecular dipole moment, where the AMOEBA force field is clearly superior (Table II). Although this is not surprising due to the lack of polarization in the rigid-ion force field, it is worth having a closer look at how the molecular dipole changes when going from the liquid to the gas phase. While the change in the water molecular dipole between the vapor (1.8D) and liquid (2.8D) phases (Fig. 4, right) can be almost entirely ascribed to the atomic polarizability, this is not the case for DCE, where a change in the relative population of the trans and gauche conformations (Table III) plays a major role. In fact, both force fields show a consistent increase in the fraction of DCE molecules in the gauche conformation when going from the gas phase to the bulk liquid, and even more when dissolved in water.
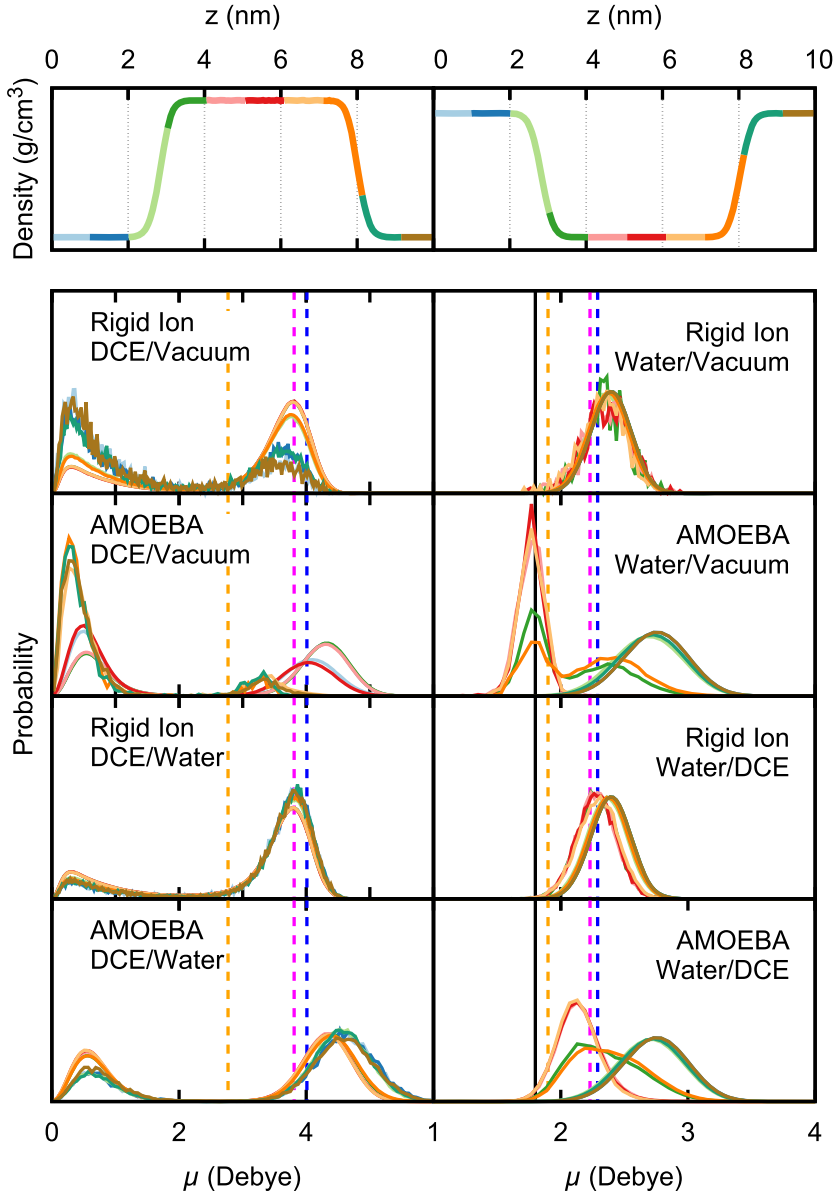

FIG. 4. Distribution of the DCE (left) and water (right) dipole moment computed in $1 \mathrm{~nm}$ slices parallel to the interface. In the top panels, a representative plot of the liquids' density is shown with each $1 \mathrm{~nm}$ slice colored differently. The same color code is used for the 10 probability distribution curves shown in the lower four panels. The top two distribution panels are for the liquid-vapor interfaces and the bottom two for the water-DCE interfaces, and they alternate between the rigid-ion and polarizable AMOEBA force fields. The black vertical line is the experimental gas phase dipole moment, and the vertical dashed lines represent the water dipole moment predicted by QM in vacuum (orange) or with an implicit solvent with parameters adequate for water (blue) or DCE (magenta)

This can be easily explained by the fact that the gauche configuration has a fairly large molecular dipole, which is stabilized by local electrostatic interactions with the surrounding polar molecules. Hence, in bulk liquid DCE, there is a cooperative stabilization effect between all the molecules in the gauche conformation; an effect that becomes even more pronounced when DCE is surrounded by molecules with a permanent dipole moment, such as water. These force field predictions were corroborated by high level QM calculations where the solvent effects are mimicked by the use of a self-consistent reaction field and are consistent with the experimental estimates obtained from IR spectroscopy. ${ }^{85}$ The prediction from the AMOEBA polarizable 
force field for the relative populations in the bulk is in much better agreement with the experiments than the rigid-ion force field, while both force fields show similar discrepancies for the vapor phase, albeit in opposite directions. Both force fields suggest an increase in the population of the gauche state when DCE is dissolved in water relative to the bulk liquid, while the QM calculations with implicit solvent performed here and QM/MM calculations ${ }^{84}$ instead predict a $10 \%$ decrease. On the other hand, the rotational barrier predicted by the rigid-ion force field appears to be three times smaller than the AMOEBA, which is instead consistent with the QM predictions and closer to the experimental estimate by Gwinn and Pitzer. ${ }^{86}$ These quantitative differences can be ascribed to a combination of polarization effects and differences in the intramolecular force field terms, and without targeted tests, it is not possible to separate the two contributions. By computing the distribution of the molecular dipole as a function of the distance from the interfaces (Fig. 4, left), it is also possible to see how these changes in the molecular conformation and the inclusion of atomic polarization combine to change the dielectric response of the liquid. It is also worth noting that at finite temperature, due to the intramolecular vibrations, even DCE molecules in the trans conformation have a relatively small dipole moment, an effect that cannot be captured in the static QM calculations.

Before moving to assess the properties of the liquid mixtures, it is worth briefly discussing the structure of the interfaces and the orientation of molecules at the liquid-vapor interfaces. The interface is still fairly sharp; however, due to the presence of capillary waves, its position varies from place to place and also with time, which gives rise to the smooth density decay over a range of $\sim 0.5 \mathrm{~nm}$ for water and $0.8 \mathrm{~nm}$ for DCE (Fig. 3). Consistent with the spectroscopic measurements and previous calculations, both force fields show that the surface water molecules are preferentially oriented with one $\mathrm{O}-\mathrm{H}$ bond pointing toward the vapor phase. ${ }^{89,90}$ It is also apparent that when moving toward regions of lower density, the polar angle of the molecular dipole decreases, which suggests that the two H-bonds to the lone pairs are the last to break before the molecule leaves the surface to go into the gas phase.

Analogous to the above results for water, DCE molecules at the liquid-vapor interfaces have a slight preference to have their dipole parallel to the normal to the interface, which suggests that chlorine atoms face toward the bulk liquid and the hydrogen atoms point toward the vapor phase. However, due to the presence of two different conformers, the situation is a bit more complicated. In this work, we have followed the conventional physics definition of the dipole vector, i.e., it goes from the negative to the positive charge.

\section{B. Properties of the water/DCE interface}

After having validated the bulk properties of the pure liquids, we turned our attention to the properties of a combined system containing both liquids together with an interface (Table IV). Immediately, it became apparent that with the standard Lorentz-Berthelot mixing rules, the rigid-ion force field was giving a positive solvation free energy for DCE in water, while the AMOEBA force field was quite close to the experimental estimate of $-7.6 \mathrm{~kJ} / \mathrm{mol}$. ${ }^{69,91}$ A full discussion regarding the comparison between the theoretical and experimental values is provided as supplementary material.
TABLE IV. Properties of the water-DCE interface. $x_{i}$ is the mole fraction of $i$ in the other solvent (see text for details).

\begin{tabular}{lccc}
\hline \hline & Expt. & Rigid-ion & AMOEBA \\
\hline$\Delta \mathrm{G}_{h y d}(\mathrm{~kJ} / \mathrm{mol})$ & $-7.6^{\mathrm{a}}$ & $-4.3 \pm 0.2$ & $-8.7 \pm 3$ \\
$\mathrm{D}_{0}\left(10^{-5} \mathrm{~cm}^{2} / \mathrm{s}\right)$ & $1.08^{\mathrm{b}}$ & $1.6 \pm 0.1$ & $1.5 \pm 0.1$ \\
$\gamma(\mathrm{dyn} / \mathrm{cm})$ & $28.3^{\mathrm{c}}$ & 31 & 30.4 \\
$10^{3} x_{D C E}$ & $1.6^{\mathrm{d}}$ & 1 & 2 \\
$10^{3} x_{\mathrm{H}_{2} \mathrm{O}}$ & $11^{\mathrm{d}}$ & 2.5 & 15 \\
$d_{0}(\mathrm{~nm})$ & $\cdots$ & 0.5 & 0.5 \\
\hline \hline
\end{tabular}

${ }^{\mathrm{a}}$ From Refs. 69 and 91.

${ }^{\mathrm{b}}$ From Ref. 94.

${ }^{\mathrm{c}}$ From Refs. 95 and 96.

${ }^{\mathrm{d}}$ From Ref. 97.

We therefore decided to re-parameterize the water-DCE force field terms to improve the solvation free energy and DCE solubility in water while keeping the properties of the pure liquids unchanged. Although not perfect, the new parameters give the correct sign for hydration free energy and diffusion coefficient of DCE in water closer to the experimental and AMOEBA values, which was deemed sufficient for the purpose of this work. Henceforth, in the remainder of this paper, we used only the force field with the refined water-DCE parameters.

In order to compute the properties of the water/DCE interface, we built a simulation cell of $5 \times 5 \times 10 \mathrm{~nm}^{3}$ with approximately a 50/50 water/DCE volume ratio. The system was oriented such that the $z$ axis was orthogonal to the interface. 3D periodic boundary conditions were used, without any vacuum gap. The system was equilibrated in the NPT ensemble by keeping the area of the interface fixed. Although the volume equilibrated fairly quickly, the equilibration was extended until the concentrations of water in DCE and DCE in water remained approximately constant to indicate that the partitioning of molecules across the interface had also reached an equilibrium distribution. In order to facilitate molecular diffusion, for these runs, we used the Nosé-Hoover thermostat. It is indeed well-known that the Langevin thermostat can significantly slow down the molecular diffusivity observed in MD simulations (see, e.g., Refs. 92 and 93). The equilibrated configurations were then used to compute the surface tension with three different cutoff radii, analogous to the liquid-vapor interfaces. The mutual solubilities of water and DCE, as well as the interface width (Fig. 5), have been computed by fitting the oxygen and chlorine density profiles using Eq. (9).

The orientation of both water and DCE at the liquid-liquid interface is qualitatively similar to the one observed at the respective liquid-vapor interfaces, although the magnitude is somewhat enhanced. In particular, we note a much stronger preference for the molecular dipole to be aligned with the $z$ axis, but antiparallel to each other (see the supplementary material). Although the conformation of the DCE molecules close to the interface is also similar to the one predicted for the liquid-vapor interface, we observe a slight increase in the fraction of the gauche conformer for the molecules that are dissolved in water. This again can be explained by the fact 


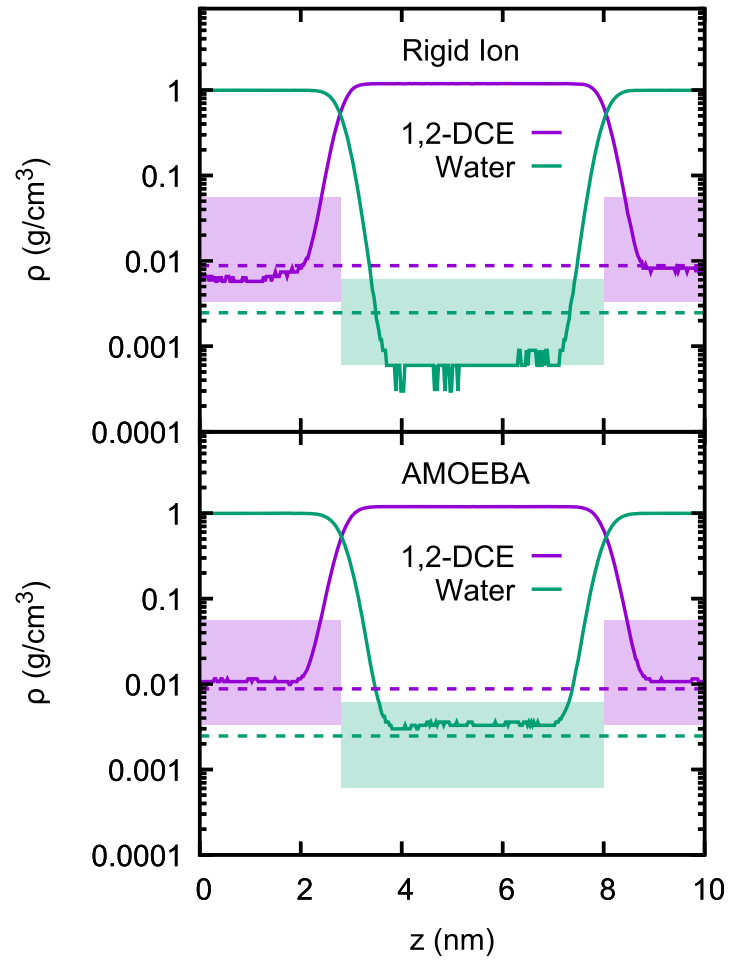

FIG. 5. Water and DCE density profiles along the direction normal to the interface (z) on a logarithmic scale. The shaded areas indicate the range of the experimental values for the solubilities of water in DCE and of DCE in water that were included in the compilation of the IUPAC-NIST Solubility Data Series, while the dashed lines are the recommended values.

that the conformer with the larger molecular dipole is stabilized by the water permanent dipoles. The structure and dynamics of the water-DCE interface observed in our simulations is consistent with the capillary wave theory and previous simulations by Willard and Chandler. ${ }^{8}$ In fact, there is no indication of mixing on a molecular level, and the water molecules, even relatively far from the nominal position of the interface, maintain a connection with the bulk liquid via the hydrogen bond network until they break free from the bulk liquid and start diffusing away into the DCE phase (see Fig. 6 and the supplementary material). Thus, for any given instantaneous configuration, the interface would appear to undulate on the nanometer scale while remaining fairly sharp at any given location. Of course, for the experimental techniques that are sensitive to the average density of molecules over a macroscopic length scale, the two pictures are hardly distinguishable. Particularly in the case of the AMOEBA force field, we note that the water-water coordination number is not exactly zero on the DCE side of the interface, which indicates that often water molecules leave the interface in small clusters, which are then preserved in DCE. However, due to the very low solubility of water in DCE, it was not possible to accumulate enough statistics on these clusters to provide quantitative information on their size distribution.

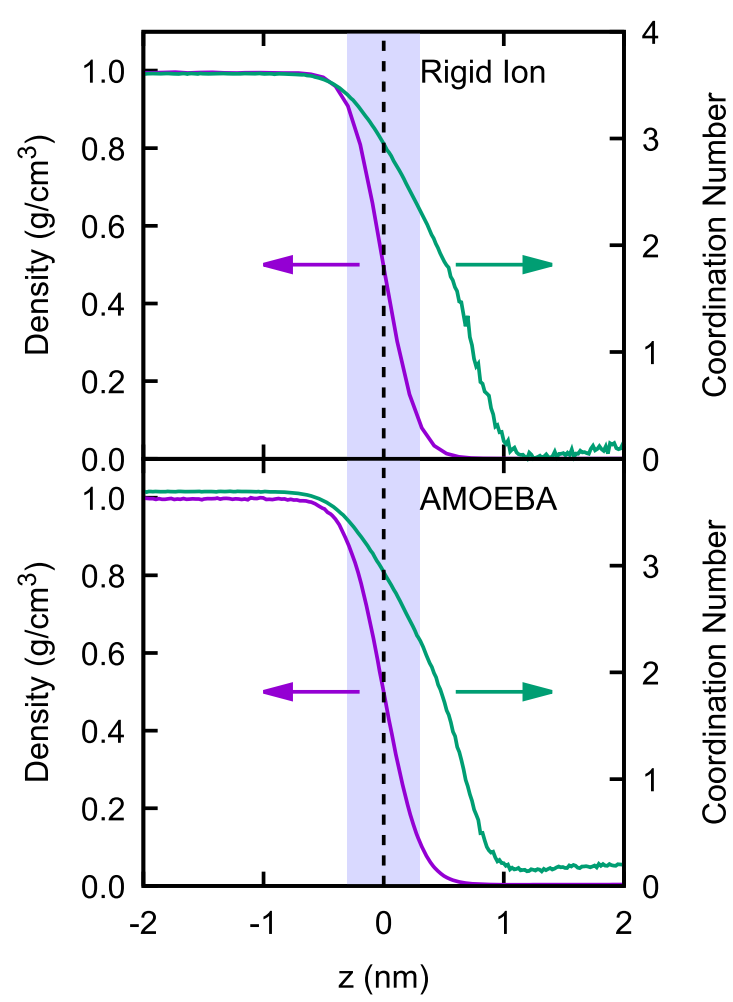

FIG. 6. Average water by water coordination number as a function of their position relative to the interface with DCE for the rigid-ion (top) and AMOEBA (bottom) force fields. The coordination number has been computed by simply counting the neighboring water molecules within a $3.1 \AA$ cutoff radius. The shaded area indicates the interface width computed by fitting the density profile with Eq. (9).

\section{APPLICATION OF AN EXTERNAL ELECTRIC FIELD}

After having validated the rigid-ion and AMOEBA force fields for the water-DCE interface, we can turn our attention to applying an external electric field to polarize the interface. Although the lack of background electrolytes makes the comparison with experiments difficult, the focus here is on the methodological side of how to correctly apply an electric field in $3 \mathrm{D}$ periodic $\mathrm{MD}$ simulations using the Ewald summation method; the addition of background electrolyte ions will be discussed in future work. As it was mentioned in the Introduction, the tin-foil boundary condition is the most commonly used implementation of the Ewald sum, and it is also the one used in the three MD codes employed in this work. In many published studies, the electric field is simply applied as a force proportional to the atomic charges across the whole simulation cell. However, this approach is often seriously flawed, if used or interpreted incorrectly, as it does not take into account the requirement under the tin-foil approximation that no permanent dipole is present in the simulation cell: In other words, it assumes that there is an equal flow of charge elsewhere in the system in the direction opposing the electric field (e.g., via an electrical circuit between the two opposing surfaces). Moreover, a simple charge proportionality does not account for the fact that in a heterogeneous system, such as a liquid-liquid interface, 
the electric field is different on either side of the interface, because of the different dielectric properties of the materials. Furthermore, in $3 \mathrm{D}$ periodic boundary conditions, there is also an ambiguity about where the interface is placed within the simulation cell, and the common approach of adding a vacuum gap and restraining the atoms to stay in the middle of the cell does not solve the problem related to the violation of the tin-foil approximation. Instead, in this work, we have designed our system as per a classical physics textbook capacitor (Fig. 2). For all the simulations reported here, a $10 \mathrm{~nm}$ thick water slab, surrounded by two $5 \mathrm{~nm}$ DCE slabs, was placed in-between two planes of point charges, and weak harmonic restraints were used to keep the molecules at least $1 \mathrm{~nm}$ away from the charged "plates" (dashed lines in Fig. 2). All simulation cells were $5 \times 5 \mathrm{~nm}^{2}$ in the $x y$ plane. The system was then replicated in the $z$ direction by taking its mirror image. The strength of the electric field was then varied by assigning different charges to the point particles that form the capacitor plates. It is immediately evident how any dipole that is induced by the external electric field cancels out in the simulation cell due to the intrinsic symmetry of the system, which makes the use of the 3D Ewald summation method possible.

For the AMOEBA polarizable force field, the electric field can only be computed from the induced dipoles $\left(\vec{\mu}_{i}\right)$,

$$
\vec{F}_{i}=\vec{\mu}_{i} / \alpha_{i}
$$

where $\alpha_{i}$ are the atomic polarizabilities; ${ }^{98}$ hence, we inserted a polarizable neutral test particle in various positions of the system. The induced and total atomic dipoles for the AMOEBA force field were obtained by post-processing the MD trajectories using an in-house code that employed the openMM libraries to compute the AMOEBA self-consistent dipolar and multipolar electrostatic energy. In order to maintain a consistency in the analysis, we decided to use the same procedure also for the rigid-ion force field, and for the postprocessing of the data, we assigned a small polarizability to each atom $\left(\alpha=10^{-4} \AA^{3}\right)$. However, to minimize any spurious effects, the induced dipoles were not optimized self-consistently, but purely determined based on the atomic charges of the rigid-ion force field, and the Thole damping of the charges was removed.

It is easy to show that for an infinite planar capacitor, there is no electric field outside the plates and that the electric field inside the plates is

$$
E \hat{\mathbf{z}}=\frac{\sigma}{\varepsilon_{0} \varepsilon_{r}} \hat{\mathbf{z}},
$$

where $\sigma$ is the charge density on the plates, $\varepsilon_{0}$ is the vacuum permittivity, $\varepsilon_{r}$ is the relative permittivity of the material, and $\hat{\mathbf{z}}$ is a unit vector normal to the charged plates. In order to verify that this is reproduced by our setup, we run a series of tests where we computed the electric field across the system in the absence of any water and DCE, i.e., $\varepsilon_{r}=1$ (Fig. 7). In this case, a neutral polarizable test particle was added in different positions in the simulation cell. For comparison, the electrostatic potential was computed on a $3 \mathrm{D}$ grid using the "pmepot" plugin" in VMD ${ }^{100}$ with a Gaussian sharpness of $1000 \AA^{-1}$. We used a grid spacing of $1 \AA$ in the $x$ and $y$ directions and of $0.25 \AA$ in the $z$ direction. The final electrostatic potential along $z$ was then computed by averaging the values in all the corresponding $x y$ planes.

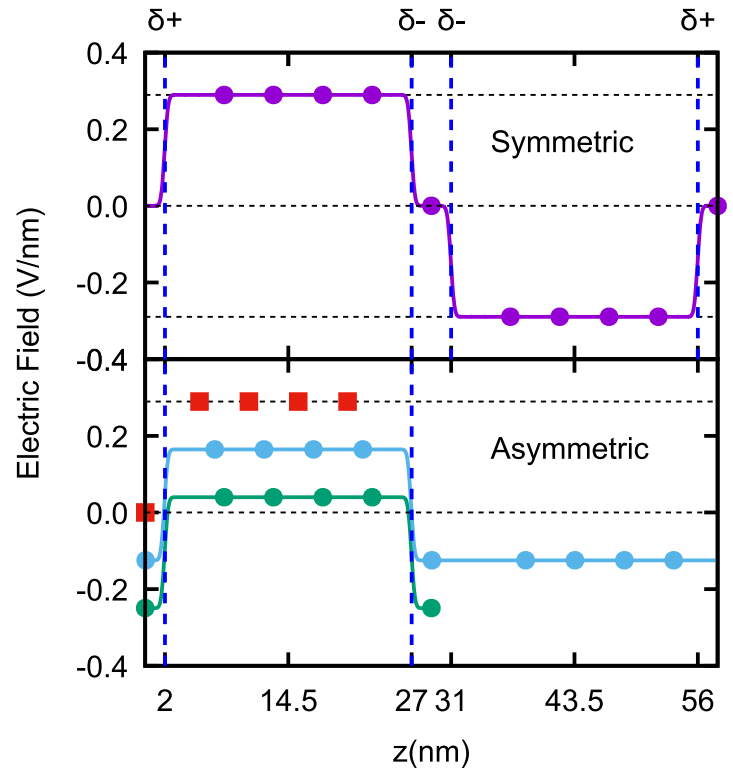

FIG. 7. Comparison between the electric field computed with the periodic 3D Ewald summation methods using the symmetric setup with four charged plates proposed here (top) and an asymmetric system with only two charges plates (bottom). The horizontal dashed lines represent the expected electric field inside $(0.29 \mathrm{v} / \mathrm{nm})$ and outside $(0 \mathrm{~V} / \mathrm{nm})$ the capacitor. The solid lines are the electric field computed for the rigid-ion model using the "pmepot" plugin in VMD, while the points were computed using Eq. (12) (circles) or the 2D Parry method with GULP (squares). The blue vertical lines show the position of the charge plates. The simulation cell was $58 \mathrm{~nm}$ along $z$ for the symmetric setup and $29 \mathrm{~nm}$ or $58 \mathrm{~nm}$ for the asymmetric setup (see text for further details).

Figure 7 demonstrates how our symmetric setup is able to reproduce the correct macroscopic electric field inside the capacitor (black solid line) when using the Ewald summation with $3 \mathrm{D}$ periodic boundary conditions both for the rigid-ion (purple line) and AMOEBA force fields (filled circles). On the other hand, when only half of the system is used with a 3D Ewald sum (green line and circles), the predicted electric field inside the capacitor is significantly different from the expected value. This is in fact due to the tin-foil approximation, which enforces a net zero electrostatic potential inside the simulation cell, and therefore, the integral of the electric field inside and outside the capacitor has to compensate each other. Hence, the actual value of the electric field inside a capacitor for the asymmetric setup depends on the relative volume of the system inside and outside the capacitor (blue and green lines). As mentioned before, there are exact ways of computing the longrange electrostatics in $2 \mathrm{D}$, such as the method proposed by Parry ${ }^{36}$ (squares), which indeed correctly reproduces the expected value of the electric field inside the atomic capacitor (squares). Finally, it is worth mentioning that due to the fact that our charged plates were simulated with a square arrangement of point particles, the lines of the electric field are not exactly perpendicular to the plates at very short distance, but this effect vanishes very rapidly, and the field can be considered uniform in the region where the water/DCE slab was kept by the harmonic restraints. 
In order to further validate our system setup, we computed the average local electric field inside a slab of pure water sandwiched between two DCE slabs (Fig. 8) using the rigid-ion force field (red) and the AMOEBA polarizable force field (blue). In accord with Eq. (13), the average local electric field computed from the rigid-ion simulations is $E_{\text {ext }} / \varepsilon_{r}$, where $\varepsilon_{r}=19$ for DCE and $\varepsilon_{r}=80$ for water. On the other hand, for the AMOEBA polarizable force field, the local electric field is significantly larger than the average macroscopic electric field in the liquids. This is because for a polarizable model, the local electric field is the sum of the macroscopic field and the field generated by the electric polarization of the material, ${ }^{101}$

$$
\left\langle E_{\text {local }}\right\rangle=E+b \frac{P}{\varepsilon_{0}},
$$

where $E=E_{\text {ext }} / \varepsilon_{r}$ is the macroscopic electric field in the dielectric medium and $P=n p$ is the electric polarization, which is proportional to the molecular polarizability, $p$, and number density, $n$, of the liquid. Hence, only for a non-polarizable force field, where $p=0$, or when the density of particles is very small (e.g., in the gas phase), the average local electric field is equal to the macroscopic field. The second term on the right-hand side of Eq. (14) corresponds to the average electric field associated with the molecular polarization inside a cavity that envelops the molecule. The parameter $b$ depends on the shape of the cavity; it is typically $\mathscr{O}(1)$ and equal to $1 / 3$ for a spherical cavity. By substituting into Eq. (14), the dependence of the electric polarizability on the electric field inside the dielectric, $P=\varepsilon_{0}\left(\varepsilon_{r}\right.$ -1) $E=\varepsilon_{0}\left(\varepsilon_{r}-1\right) E / \varepsilon_{r}$, we can rewrite the average local electric field in terms of the vacuum field, $E_{\text {ext }}$, which can be easily computed from the charge density on the plates of the capacitor,

$$
\left\langle E_{\text {local }}\right\rangle=\frac{1+b\left(\varepsilon_{r}-1\right)}{\varepsilon_{r}} E_{\text {ext }} .
$$

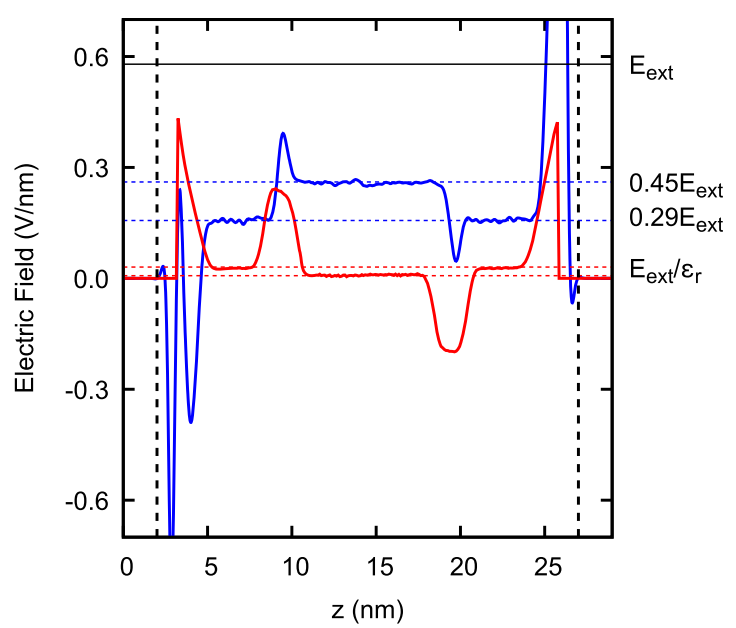

FIG. 8. Comparison between the local electric field in the water/DCE slab predicted by the rigid-ion (red) and AMOEBA (blue) force fields. The horizontal lines are the expected values for the rigid-ion force field and the best fit lines of the flat regions for the AMOEBA force field (see text for more details).
For a spherical cavity, where $b=1 / 3,\left\langle E_{\text {local }}\right\rangle / E_{\text {ext }} \approx 0.35$, in good agreement with the values predicted by the AMOEBA polarizable force field for both water (0.45) and DCE (0.29). Moreover, Fig. 8 clearly shows that the strength of the local electric field is significantly different in water and DCE, which further emphasizes how the use of a force proportional to the atomic charges is not a proper way to apply an external electric field to an inhomogeneous system. Although different forces could be applied to different species, this requires knowing a priori the dielectric constants of the simulated materials and completely disregards the fact that the bulk value of the dielectric constant may not be appropriate for the interfacial regions. This approach becomes even more problematic for liquidliquid interfaces, which are not flat, and where the species partially mix.

The presence of an external electric field has a limited influence on the structure of the water/DCE interface, and in all cases, the width of the interface remains constant at $\sim 5 \AA$. The orientation of water's molecular plane and the relative population of the trans and gauche conformations in bulk DCE also remain unchanged (see the supplementary material). Although, surprisingly, the distribution of the values of the molecular dipoles is also unaffected by the presence of an external field, there is a significant difference in the average orientation of the molecular dipoles. It is well-known that in polar liquids, such as water, the presence of an electric field induces a preferential orientation of the dipoles parallel to the direction of the field. For weak electric fields, the degree of alignment is linear with the field. Although, in the case of freely rotating dipoles, the proportionality constant was analytically derived more than century ago by Onsager and Kirkwood, ${ }^{102,103}$ for real liquids, its exact value is generally an unknown quantity. It is easy to see that the degree of alignment should depend on the inverse of the temperature, which tends to randomize the dipoles' orientation, and on magnitude of the molecular dipole itself, as larger dipoles are subject to stronger torques. Hence, we can generalize the Kirkwood-Onsager formula as

$$
\langle\cos \theta\rangle=\alpha \frac{\mu_{0}}{k_{B} T} \frac{E_{e x t}}{\varepsilon_{r}},
$$

where all the unknown quantities related to the particular molecular structure of the liquids have been included in the fitting parameter $\alpha$ and $\mu_{0}$ is taken as the average molecular dipole. In agreement with Eq. (16), the average orientation of the molecular dipoles predicted by the rigid-ion and AMOEBA force fields increases linearly with the external electric field field (Fig. 9). The rigid-ion and AMOEBA polarizable force fields predict very similar degrees of orientation of the molecular dipoles across the whole range of electric fields explored here, which agrees with the fact that the two models have similar dielectric constants for the liquids, and therefore, the macroscopic electric field in the media should be the same. Moreover, the slope observed for the dipole of DCE is much larger than that for water, in accord with the fact that the macroscopic electric field in DCE is much larger than in water due to its dielectric constant being approximately 4 times smaller.

It is also interesting to note here that the presence of a weak electric field has a negligible effect on the distribution of the molecular dipoles, both for DCE and water (see the supplementary material). Moreover, for the species studied in this work, the ratio 


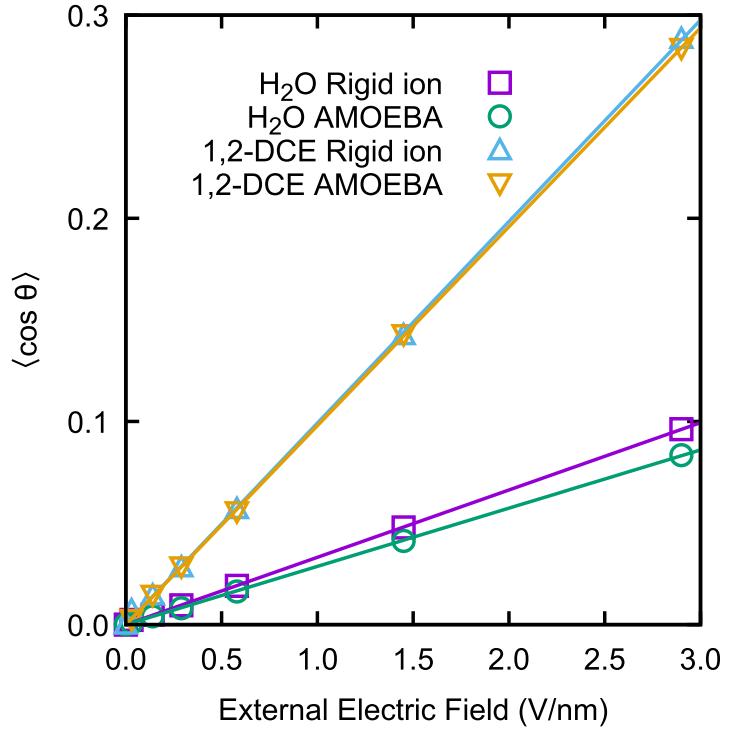

FIG. 9. Average orientation of the molecular dipoles relative to the direction of the electric field $(\langle\cos \theta\rangle)$ computed with the rigid-ion and AMOEBA force fields.

$\mu_{0} / \varepsilon_{r}$ is independent of the choice of force field. Hence, the slight differences in the slopes shown in Fig. 9 can be ascribed to differences in the intermolecular interactions between the rigid-ion and AMOEBA force fields.

\section{DISCUSSION}

Finally, it is important to discuss how the method proposed here of applying an electric field using two sets of oppositely charged plates compares to other approaches commonly found in the literature, and to the use of only one set of charged plates. In order to do this, we simulated a DCE/water/DCE slab under a uniform electric field parallel to the $z$ axis using the rigid-ion force field. The electric field was applied either via charged plates using both the asymmetric and symmetric setups or applying a force to each atom that was proportional to their charge, $F=q E$. In order to make the comparison more quantitative, for the simulations without the charged plates, we chose the electric field to be $E=E_{\text {ext }} / \varepsilon_{r}$, where $E_{\text {ext }}$ is the field generated by the charged plates in the vacuum region outside the slab and $\varepsilon_{r}=80$ is the dielectric constant of SPC/Fw water. For these simulations, the local electric field was defined as the field generated by the atoms plus the applied field. Figure 10 clearly shows that the symmetric setup proposed here is the only method that correctly reproduces the expected magnitude of the electric field on both sides of the DCE and water interface (dashed lines), which is also reflected in the different average orientations of the molecular dipoles predicted by the different approaches. Although we do not have a way to directly compute the local electric field with the slab correction proposed by Yeh and Berkowitz, ${ }^{32}$ which is implemented in LAMMPS via the "slab" keyword, we note that the average orientation of the molecular dipoles with the "asymmetric" setup is almost identical to the one predicted by the "symmetric" simulation

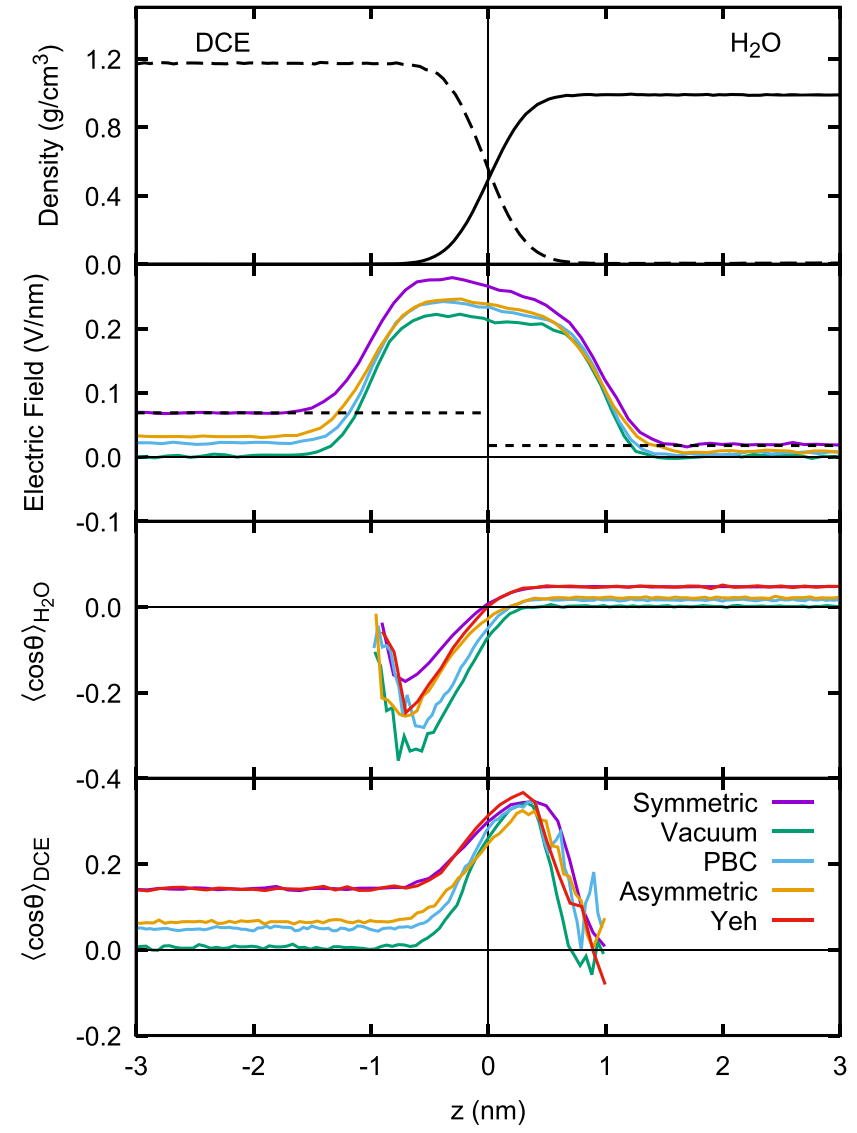

FIG. 10. From top to bottom, comparison between the water and DCE density, electric field, water dipole average orientation, and DCE dipole average orientation in the presence of an external electric field for different simulation setups obtained with the rigid-ion force field (Fig. 2). The electric field was applied as a force proportional to the atomic partial charges for the "vacuum" and "PBC" setups. The asymmetric setup was also run with the correction term proposed by Yeh and Berkowitz $^{32}$ (red curve labeled "Yeh"). All the curves have been aligned to the position of their respective interfaces. The black dashed horizontal lines in the electric field panel represent the expected value of the electric field in the water and DCE slabs based on the charge density on the plates and the liquids' dielectric constants.

in regions where there are statistically significant data (i.e., excluding where small numbers of molecules diffuse into the other liquid). This would indeed suggest that the electric field both in the water and DCE phases has the correct magnitude (Fig. 10).

In the case of ITIES, the dissolved electrolyte ions would diffuse parallel to the field lines and produce a charge separation, which neutralize the field in the bulk liquids, and because the electrolyte ions cannot easily cross the interface, they would accumulate there and contribute to the polarization of the interfaces itself. The magnitude of this charge separation is however proportional to the electric field, and therefore, an incorrect simulation setup would generate a quantitatively wrong estimate of the ion concentrations at the interface and of the field across the interface. 
Specifically, for the DCE-water interface, a recent work by Gschwend et al. ${ }^{104}$ studied the polarization of the water/DCE interface by applying a constant electric field in their simulation ("vacuum" setup) and also by creating an excess of charge in one phase, compensated by an equal and opposite charge in the other phase. While the first approach suffers with the problems discussed above, the second method represents an unphysical system. This is because experimentally each electrolyte is charge neutral before being put into contact with each other, and there cannot be a charge imbalance in the two immiscible liquids in the absence of an external electric field. The simulations indeed showed the expected results that the ions swiftly migrated toward the interfaces and formed strong ion pairs to neutralize the charge imbalance in the system, and if either of the ions was soluble in the other phase, they would have spontaneously crossed the interface and created two neutral electrolytes. However, in a realistic experimental setup, where both electrolytes are charge neutral, there can be a significant accumulation of ions at the interface only in the presence of an electric field, and because the strength of the field depends on the dielectric constant of the medium, there is no guarantee that the same amount of charge would accumulate on both sides of the interface.

\section{CONCLUSIONS}

In this study, we describe a simple geometric method to correctly apply an external electric field in 3D periodic MD simulations, which is compatible with standard 3D Ewald summation algorithms. The electric field is applied by using two sets of oppositely charged plates surrounding the two copies of the atomistic system, which mimic two parallel plate capacitors with a dielectric. Because of the intrinsic symmetry of our setup, any macroscopic dipole that is induced by the electric field cancels out in the simulation cell, on average, which makes it compatible with the tinfoil approximation that is normally used in 3D Ewald summation methods.

This method was tested on the interface between two immiscible liquids, water and 1,2-dichloroethane, using both a rigid-ion and a polarizable force field. The former was based on the GAFF force field, while the latter was newly developed in this work within the AMOEBA framework. Both force fields were thoroughly tested to ensure that they correctly reproduced the thermodynamics properties of the liquid and of the binary system. The structure of both the liquid-vapor and liquid-liquid interfaces was also shown to be consistent with the experimental molecular orientations inferred from spectroscopic measurements.

By changing the charge density on the capacitors' plates, we showed that the magnitude of the electric field inside the two liquids is different and proportional to their respective dielectric constants, in agreement with the expected macroscopic behavior of dielectrics. By comparing the results from our symmetric simulation cell with other system designs commonly found in the literature, we demonstrated that approximating the presence of an external electric field with a force proportional to the atomic charges would produce the wrong dielectric response in the liquids. Our results also show that a simple slab geometry, where the atomistic region is separated from its periodic images by a vacuum region, is a necessary, but not sufficient, condition for reproducing the correct behavior of materials in the presence of an external electric field, if the long range electrostatic is computed using the standard 3D Ewald summation method.

\section{SUPPLEMENTARY MATERIAL}

See the supplementary material for all the force field parameters, for a discussion about the comparison of the calculated solvation free energies with the experimental values, for the distributions of the DCE torsional angle at the liquid-vacuum and liquid-liquid interfaces, for a comparison of the electric field computed with VMD and via the atomic induced dipoles, for the electric field inside water and DCE as a function of the strength of the external field, and for the distributions of the magnitude and orientation of the molecular dipoles with different external electric fields predicted by the rigid-ion and AMOEBA force fields.

\section{ACKNOWLEDGMENTS}

The computational resources have been provided by the Australian Government and the Government of Western Australia through the Pawsey Supercomputing Centre and the National Computational Infrastructure under the National Computational Merit Allocation Scheme. J.D.G. thanks the Australian Research Council for funding under Grant No. FL180100087. P.K. would like to thank the Forrest Research Foundation for his fellowship. The authors would also like to thank Professor Damien Arrigan for useful discussions.

\section{DATA AVAILABILITY}

The data that support the findings of this study are available from the corresponding author upon reasonable request.

\section{REFERENCES}

${ }^{1}$ A. G. Volkov, Liquid Interfaces in Chemical, Biological and Pharmaceutical Applications (Dekker, Abingdon, 2001).

${ }^{2}$ T. Sato, Hydrometallurgy 22, 121 (1989).

${ }^{3}$ Z. Samec, Pure Appl. Chem. 76, 2147 (2004).

${ }^{4}$ H. H. Girault, in Electroanalytical Chemistry A Series of Advances, edited by A. J. Bard and C. G. Zoski (CRC Press, 2010), Vol. 23, pp. 1-104.

${ }^{5}$ D. W. M. Arrigan, G. Herzog, M. D. Scanlon, and J. Stutwolf, in Electroanalytical Chemistry A Series of Advances, edited by A. J. Bard and C. G. Zoski (CRC Press, 2019), Vol. 25, pp. 105-178.

${ }^{6}$ F. P. Buff, R. A. Lovett, and F. H. Stillinger, Phys. Rev. Lett. 15, 621 (1965).

${ }^{7}$ E. H. Lucassen-Reynders and J. Lucassen, Adv. Colloid Interface Sci. 2, 347 (1970).

${ }^{8}$ A. P. Willard and D. Chandler, J. Phys. Chem. B 114, 1954 (2010).

${ }^{9}$ H. H. Girault and D. J. Schiffrin, J. Electroanal. Chem. Interfacial Electrochem. 150, 43 (1983).

${ }^{10}$ D. S. Walker, M. G. Brown, C. L. McFearin, and G. L. Richmond, J. Phys. Chem. B 108, 2111 (2004).

${ }^{11}$ D. S. Walker and G. L. Richmond, J. Am. Chem. Soc. 129, 9446 (2007). 
${ }^{12}$ S. Ishizaka, S. Habuchi, H.-B. Kim, and N. Kitamura, Anal. Chem. 71, 3382 (1999).

${ }^{13}$ J. G. Frey, in Interfacial Nanochemistry: Molecular Science and Engineering at Liquid-Liquid Interfaces, edited by H. Watarai, N. Teramae, and T. Sawada (Springer US, Boston, MA, 2005), pp. 1-23.

${ }^{14}$ P. S. Pershan, Physica A 231, 111 (1996).

${ }^{15}$ M. L. Schlossman, Curr. Opin. Colloid Interface Sci. 7, 235 (2002).

${ }^{16}$ I. Benjamin, J. Chem. Phys. 97, 1432 (1992).

${ }^{17}$ I. Benjamin, Science 261, 1558 (1993).

${ }^{18}$ I. Benjamin, Annu. Rev. Phys. Chem. 48, 407 (1997).

${ }^{19}$ G. Hantal, P. Terleczky, G. Horvai, L. Nyulászi, and P. Jedlovszky, J. Phys. Chem. C 113, 19263 (2009).

${ }^{20}$ D. J. V. A. dos Santos and J. A. N. F. Gomes, Chemphyschem 3, 946 (2002).

${ }^{21}$ N. Holmberg, M. Sammalkorpi, and K. Laasonen, J. Phys. Chem. B 118, 5957 (2014).

${ }^{22}$ J. J. Karnes and I. Benjamin, J. Chem. Phys. 145, 014701 (2016).

${ }^{23}$ I. Benjamin, J. Chem. Phys. 151, 094701 (2019).

${ }^{24}$ B. S. Dwadasi, S. Goverapet Srinivasan, and B. Rai, Phys. Chem. Chem. Phys. 22, 4177 (2020).

${ }^{25}$ K. J. Schweighofer and I. Benjamin, J. Electroanal. Chem. 391, 1 (1995).

${ }^{26}$ G. Luo, S. Malkova, J. Yoon, D. G. Schultz, B. Lin, M. Meron, I. Benjamin, P. Vanysek, and M. L. Schlossman, Science 311, 216 (2006).

${ }^{27}$ N. Laanait, M. Mihaylov, B. Hou, H. Yu, P. Vanysek, M. Meron, B. Lin, I. Benjamin, and M. L. Schlossman, Proc. Natl. Acad. Sci. U. S. A. 109, 20326 (2012).

${ }^{28}$ D. Frenkel and B. Smit, Understanding Molecular Simulation, From Algorithms to Applications (Academic Press, 2002).

${ }^{29}$ I.-C. Yeh and G. Hummer, J. Phys. Chem. B 108, 15873 (2004).

${ }^{30}$ L. Lundberg and O. Edholm, J. Chem. Theory Comput. 12, 4025 (2016).

${ }^{31}$ S. Bogusz, T. E. Cheatham III, and B. R. Brooks, J. Chem. Phys. 108, 7070 (1998).

${ }^{32}$ I.-C. Yeh and M. L. Berkowitz, J. Chem. Phys. 111, 3155 (1999).

${ }^{33}$ W. Weber, P. H. Hünenberger, and J. A. McCammon, J. Phys. Chem. B 104, 3668 (2000).

${ }^{34}$ E. Spohr, J. Chem. Phys. 107, 6342 (1997).

${ }^{35}$ S. de Leeuw, J. Perram, and E. Smith, Proc. R. Soc. London, Ser. A 373, 27-56 (1980).

${ }^{36}$ D. E. Parry, Surf. Sci. 49, 433 (1975).

${ }^{37}$ D. E. Parry, Surface Science 54, 195 (1976).

${ }^{38}$ P. E. Smith, H. D. Blatt, and B. M. Pettitt, J. Phys. Chem. B 101, 3886 (1997).

${ }^{39}$ J. S. Hub, B. L. de Groot, H. Grubmüller, and G. Groenhof, J. Chem. Theory Comput. 10, 381 (2014).

${ }^{40}$ C. Caleman, P. J. van Maaren, M. Hong, J. S. Hub, L. T. Costa, and D. van der Spoel, J. Chem. Theory Comput. 8, 61 (2011).

${ }^{41}$ S. Plimpton, J. Comput. Phys. 117, 1 (1995).

${ }^{42}$ L. Lagardère, L.-H. Jolly, F. Lipparini, F. Aviat, B. Stamm, Z. F. Jing, M. Harger, H. Torabifard, G. A. Cisneros, M. J. Schnieders, N. Gresh, Y. Maday, P. Y. Ren, J. W. Ponder, and J.-P. Piquemal, Chem. Sci. 9, 956 (2018).

${ }^{43}$ P. Eastman and V. S. Pande, J. Comput. Chem. 31, 1268 (2010).

${ }^{44}$ P. Eastman, J. Swails, J. D. Chodera, R. T. McGibbon, Y. Zhao, K. A. Beauchamp, L.-P. Wang, A. C. Simmonett, M. P. Harrigan, C. D. Stern, R. P. Wiewiora, B. R. Brooks, and V. S. Pande, PLoS Comput. Biol. 13, e1005659 (2017).

${ }^{45}$ J. Wang, R. M. Wolf, J. W. Caldwell, P. A. Kollman, and D. A. Case, J. Comput. Chem. 25, 1157 (2004).

${ }^{46}$ Y. Wu, H. L. Tepper, and G. A. Voth, J. Chem. Phys. 124, 024503 (2006).

${ }^{47}$ M. L. Laury, L.-P. Wang, V. S. Pande, T. Head-Gordon, and J. W. Ponder, J. Phys. Chem. B 119, 9423 (2015).

${ }^{48}$ J. C. Wu, G. Chattree, and P. Ren, Theor. Chem. Acc. 131, 107 (2012).

${ }^{49}$ A. J. Stone, J. Chem. Theory Comput. 1, 1128 (2005).

${ }^{50}$ J. W. Ponder, C. Wu, P. Ren, V. S. Pande, J. D. Chodera, M. J. Schnieders, I. Haque, D. L. Mobley, D. S. Lambrecht, R. A. DiStasio, M. Head-Gordon, G. N. I. Clark, M. E. Johnson, and T. Head-Gordon, J. Phys. Chem. B 114, 2549 (2010).
${ }^{51}$ R. W. Hockney, S. P. Goel, and J. W. Eastwood, Chem. Phys. Lett. 21, 589 (1973).

${ }^{52} \mathrm{~J}$. W. Eastwood, R. W. Hockney, and D. N. Lawrence, Comput. Phys. Commun. 19, 215 (1980).

${ }^{53}$ U. Essmann, L. Perera, M. L. Berkowitz, T. Darden, H. Lee, and L. G. Pedersen, J. Chem. Phys. 103, 8577 (1995).

${ }^{54}$ T. Darden, D. York, and L. Pedersen, J. Chem. Phys. 98, 10089 (1993).

${ }^{55}$ J. D. Gale and A. L. Rohl, Mol. Simul. 29, 291 (2003).

${ }^{56}$ J. D. Gale, Z. Kristallogr. 220, 552 (2005).

${ }^{57}$ P. Raiteri, A. Schuitemaker, and J. D. Gale, J. Phys. Chem. B 124, 3568 (2020).

${ }^{58}$ E. H. Byrne, P. Raiteri, and J. D. Gale, J. Phys. Chem. C 121, 25956 (2017).

${ }^{59}$ D. G. A. Smith, L. A. Burns, A. C. Simmonett, R. M. Parrish, M. C. Schieber, R. Galvelis, P. Kraus, H. Kruse, R. Di Remigio, A. Alenaizan, A. M. James, S. Lehtola, J. P. Misiewicz, M. Scheurer, R. A. Shaw, J. B. Schriber, Y. Xie, Z. L. Glick, D. A. Sirianni, J. S. O’Brien, J. M. Waldrop, A. Kumar, E. G. Hohenstein, B. P. Pritchard, B. R. Brooks, H. F. Schaefer, A. Y. Sokolov, K. Patkowski, A. E. DePrince, U. Bozkaya, R. A. King, F. A. Evangelista, J. M. Turney, T. D. Crawford, and C. D. Sherrill, J. Chem. Phys. 152, 184108 (2020).

${ }^{60}$ L. Goerigk and S. Grimme, J. Chem. Theory Comput. 7, 291 (2011).

${ }^{61}$ D. Hait and M. Head-Gordon, J. Chem. Theory Comput. 14, 1969 (2018).

${ }^{62}$ F. Jensen, J. Chem. Phys. 117, 9234 (2002).

${ }^{63}$ Y. Zhao and D. G. Truhlar, Theor. Chem. Acc. 120, 215 (2008).

${ }^{64}$ R. D. Remigio, L. Frediani, A. H. Steindal, R. Bast, T. D. Crawford, L. A. Burns, W. Poelmans, and V. Weijo (2019). "PCMSolver," Zenodo. https://zenodo.org/ record/11910\#.X5Ieli8Rokg

${ }^{65}$ S. Grimme, J. G. Brandenburg, C. Bannwarth, and A. Hansen, J. Chem. Phys. 143, 054107 (2015).

${ }^{66}$ W. Wagner and A. Pruß, J. Phys. Chem. Ref. Data 31, 387 (2002).

${ }^{67}$ M. Holz, S. R. Heil, and A. Sacco, Phys. Chem. Chem. Phys. 2, 4740 (2000).

${ }^{68}$ K. R. Harris and L. A. Woolf, J. Chem. Eng. Data 49, 1064 (2004).

${ }^{69}$ A. Ben-Naim and Y. Marcus, J. Chem. Phys. 81, 2016 (1984).

${ }^{70}$ J. J. Jasper, J. Phys. Chem. Ref. Data 1, 841 (2009).

${ }^{71}$ D. G. Archer and P. Wang, J. Phys. Chem. Ref. Data 19, 371 (1990).

${ }^{72}$ G. Sivaramprasad, M. V. Rao, and D. H. L. Prasad, J. Chem. Eng. Data 35, 122 (1990).

${ }^{73}$ R. Malhotra, W. E. Price, L. A. Woolf, and A. J. Easteal, Int. J. Thermophys. 11, 835 (1990).

${ }^{74}$ D. J. Prakash, D. S. Lakshmi, M. V. Rao, and D. H. L. Prasad, Taylor Francis 33, 249 (1996).

${ }^{75}$ National Institute of Standards and TUS, NIST Chemistry Webbook: NIST Standard Reference Database Number 69 (NIST, 2000).

${ }^{76}$ F. Corradini, A. Marchetti, M. Tagliazucchi, and L. Tassi, Fluid Phase Equilib. 124, 209 (1996).

${ }^{77}$ A. D. Buckingham and L. E. Sutton, Proc. R. Soc. London, Ser. A 238, 235 (1956).

${ }^{78}$ J. Kolafa and L. Viererblová, J. Chem. Theory Comput. 10, 1468 (2014).

${ }^{79}$ N. M. Putintsev and D. N. Putintsev, Russ. J. Phys. Chem. A 85, 1113 (2011).

${ }^{80}$ S. A. Clough, Y. Beers, G. P. Klein, and L. S. Rothman, J. Chem. Phys. 59, 2254 (1973).

${ }^{81}$ Y. S. Badyal, M.-L. Saboungi, D. L. Price, S. D. Shastri, D. R. Haeffner, and A. K. Soper, J. Chem. Phys. 112, 9206 (2000).

${ }^{82}$ J. E. Mark and C. Sutton, J. Am. Chem. Soc. 94, 1083 (1972).

${ }^{83}$ E. Bock and E. Tomchuk, Can. J. Chem. 47, 4635 (1969).

${ }^{84}$ N. A. Murugan, H. W. Hugosson, and H. Ågren, J. Phys. Chem. B 112, 14673 (2008).

${ }^{85}$ K. Tanabe, Spectrochim. Acta Part A: Mol. Spectrosc. 28, 407 (1972).

${ }^{86}$ W. D. Gwinn and K. S. Pitzer, J. Chem. Phys. 16, 303 (1948).

${ }^{87}$ P. K. Yuet and D. Blankschtein, J. Phys. Chem. B 114, 13786 (2010).

${ }^{88}$ P. Raiteri, R. Demichelis, and J. D. Gale, J. Phys. Chem. C 119, 24447 (2015).

${ }^{89}$ Q. Du, R. Superfine, E. Freysz, and Y. R. Shen, Phys. Rev. Lett. 70, 2313 (1993). 
${ }^{90}$ S. Nihonyanagi, T. Ishiyama, T.-k. Lee, S. Yamaguchi, M. Bonn, A. Morita, and T. Tahara, J. Am. Chem. Soc. 133, 16875 (2011).

${ }^{91}$ M. H. Abraham, G. S. Whiting, R. Fuchs, and E. J. Chambers, J. Chem. Soc., Perkin Trans. 2, 291 (1990).

${ }^{92}$ J. E. Basconi and M. R. Shirts, J. Chem. Theory Comput. 9, 2887 (2013).

${ }^{93} \mathrm{~K}$. Gaalswyk, E. Awoonor-Williams, and C. N. Rowley, J. Chem. Theory Comput. 12, 5609 (2016).

${ }^{94}$ C. L. Yaws, Yaws' Handbook of Thermodynamic and Physical Properties of Chemical Compounds (Knovel, 2003).

${ }^{95}$ B. Hou, N. Laanait, H. Yu, W. Bu, J. Yoon, B. Lin, M. Meron, G. Luo, P. Vanysek, and M. L. Schlossman, J. Phys. Chem. B 117, 5365 (2013).

${ }^{96}$ H. M. Backes, M. Jing Jun, E. Bender, and G. Maurer, Chem. Eng. Sci. 45, 275 (1990).
${ }^{97}$ A. L. Horvath, F. W. Getzen, and Z. Maczynska, J. Phys. Chem. Ref. Data 28, 395 (1999).

${ }^{98}$ S. D. Fried, L.-P. Wang, S. G. Boxer, P. Ren, and V. S. Pande, J. Phys. Chem. B 117,16236 (2013).

${ }^{99}$ A. Aksimentiev and K. Schulten, Biophys. J. 88, 3745 (2005).

${ }^{100} \mathrm{~W}$. Humphrey, A. Dalke, and K. Schulten, J. Mol. Graphics 14, 33 (1996).

${ }^{101}$ D. J. Griffiths, Introduction to Electrodynamics, 4th ed. (Pearson, Boston, MA, 2013).

${ }^{102}$ L. Onsager, J. Am. Chem. Soc. 58, 1486 (1936).

${ }^{103}$ J. G. Kirkwood, J. Chem. Phys. 7, 911 (1939).

${ }^{104}$ G. C. Gschwend, A. Olaya, and H. H. Girault, Chem. Sci. 11(39), 10807-10813 (2020). 